\title{
Proteomics approaches to identify tumor antigen directed autoantibodies as cancer biomarkers
}

\author{
Yuji Imafuku, Gilbert S. Omenn and Samir Hanash* \\ Departments of Pediatrics, Medicine, and Human Genetics, University of Michigan, Ann Arbor, MI 48109, USA
}

\begin{abstract}
The identification of autoantibodies to tumor cell proteins by proteomics approaches has great potential impact on cancer biomarker discovery. The humoral immune response represents a form of biological amplification of signals that are otherwise weak due to very low concentrations of antigen, especially in the early stages of cancers. In addition, proteomics can detect immunoreactivity directed against protein post-translational modifications. Two-dimensional gel based Western blots, protein antigen microarrays, and multiplex ELISA reactions have been applied by our group to antigen based biomarker detection and validation. The latter two are based on liquid-phase separations that are suitable for automation. This work has resulted in the identification of numerous cancer biomarker candidates. Large clinical studies are currently planned to establish their value in early cancer diagnosis.
\end{abstract}

Keywords: Proteomics, autoantibodies, 2D Western blot, protein microarray, ELISA

\section{Introduction}

The early detection of cancer, as for other diseases, is one of the most important and challenging endeavors in clinical medicine. Blood, the most accessible tissue, may contain molecular indicators of the presence of cancer in various organs. Current serum based cancer biomarkers have limited utility to detect cancer early.

Proteomics approaches are emerging as useful means to discover potential cancer biomarkers [7]. Various approaches are presented in this special issue of the journal. Cancer-specific autoantibodies are especially promising as means for early diagnosis. The amount of any cancer-specific antigen in tumor cells or in the circulation is usually very low, especially in the early stage of cancer. The body's immune response to such antigens represents a remarkable phenomenon of bio-

*Corresponding author: Dr. Samir Hanash, Department of Pediatrics, University of Michigan, Room A520, MSRB I, $1150 \mathrm{~W}$. Medical Center Drive, Ann Arbor, MI 48109-0656, USA. Tel.: +1 734763 9311; Fax: +1 734647 8148; E-mail: shanash@umich.edu. logical amplification of these weak signals from cancerspecific antigens [8].

Autoantibody responses to tumors are thought to be elicited by over-expression of particular proteins, especially on the cell surface; by modification of proteins revealing new epitopes as immunogens; and by release or secretion of such normal, mutated, cleaved, or otherwise modified proteins. $\mathrm{p} 53$ is an example of mutated proteins [23]. In contrast, Crt32, a truncated form of calreticulin, reported to elicit a humoral response in hepatocellular carcinoma (HCC) patients, was found overexpressed in HCC tumor tissue compared to normal liver tissue [14]. Though calreticulin elicits a humoral response also in autoimmune diseases like systemic lupus erythematosus (SLE), the detailed epitope (N-terminal) in SLE is different from the one recognized in HCC patients (C-terminal), suggesting different mechanisms to elicit autoimmune response between tumor patients and autoimmune disease patients [14]. Two kinds of ubiquitin C-terminal hydrolase were reported to elicit humoral responses in lung and colon cancer patients. Isozyme 1 (UCH-L1, PGP 9.5), which elicited a humoral response in lung cancer 
patients, is widely expressed in neuronal tissues at all stages of neuronal differentiation and also in lung cancers [3]. Isozyme 3 (UCH-L3) is highly expressed in colon cancer [18]. These examples of cancer-specific autoantibodies are candidate cancer biomarkers. Other investigators have identified humoral responses against cancer-specific antigens by screening cDNA expression libraries $[4,13,19]$ or random peptide libraries [17].

\section{Proteomic approaches applicable to the identification of autoantibodies to tumor antigens}

Proteomics approaches are ideal for seeking cancerspecific antigens and corresponding autoantibodies. It is feasible to detect antibodies reacting to proteins with post-translational modifications, which cannot be detected using the recombinant protein methods [8]. 2D Western blots have been used as the standard method. Diluted patient sera are reacted with proteins, typically derived from cancer cell line lysates (see Section 3). More recently, protein microarrays have been employed, with robotic spotting of slides with either antigens (typically from highly fractionated tumor or cell line lysates) or antibodies (commercially-available or antibodies raised against proteins of interest) [1,16, 18,21]. Table 1 lists 16 antigens identified by proteomics approaches, 15 by $2 \mathrm{D}$ gel Western blots from 8 different tumor types. The percentages of patient sera that are positive for antibodies against these antigens range from $11 \%$ to $58 \%$; thus, there is great interest in developing combinations or panels of such biomarkers for each kind of cancer or each carcinogenic mechanism. For example, $60 \%$ of lung adenocarcinoma patients' sera showed anti-annexin I or II antibodies (40\% and $37 \%$ to each, respectively) with no positive sera from healthy subjects, smokers, or patients with non-malignant lung disease [2]. $70 \%$ of HCC patients' sera showed at least one antibody out of 8 candidate biomarkers (11\% to $27 \%$ for each autoantibody) [14].

\section{Antigen identification by 2D Western blotting}

In the usual 2D Western blot approach, proteins from cancer cell lysates are separated according to charge and molecular weight by 2D-PAGE, followed by transfer to a suitable membrane and successive reactions with patient sera and then (after washing) with enzymeconjugated anti-human IgG antibody. Chemilumines- cence is used to monitor development of the blot. After the comparison of blots with sera from cancer patients versus sera from normal individuals, cancer-associated spots are cut out, digested, and analyzed by mass spectrometry to identify the protein(s). For the purpose of comparison of blots, we transform analogue data of blots containing the position and the intensity (density) into digital quantitative or semi-quantitative data by scanner or CCD camera and use specific software to identify blotted proteins in the same position in different films and their differences of signal. Specific autoantibodies have been discovered with Western blots in sera of patients with various cancers, including antibodies to annexin I, annexin II, and PGP 9.5 (UCHL1) in lung cancers [2,3], vimentin and calreticulin in pancreatic adenocarcinoma $[9,10], \beta$-tubulin in neuroblastoma [20], RS/DJ-1 in breast cancer [15], calreticulin and others in HCC [14], and smooth muscle protein 22- $\alpha$ (SM22- $\alpha$ ) and carbonic anhydrase I in renal cell carcinoma (RCC) [12]. These latter two proteins were specifically identified by Edman sequencing among 5 spots initially recognized by RCC patients' sera compared to normal sera. Very preliminary data with sera obtained up to 12 months before the diagnosis of lung cancer indicate that at least some individuals are autoantibody-positive well before there are clinical signs of cancer (Omenn, Hanash, unpublished). Extensive follow-on studies with beta-Carotene and Retinol Efficacy Trial (CARET) specimens and with sera from participants in the spiral CT lung cancer screening studies are being organized. Although this 2D Western approach is now a standard method, it has distinct limitations in the detection of low abundance proteins and is not well suited to automation. However, compared with antigenic proteins from cancer cells expressed in E. coli using cDNA expression libraries [13], 2D gels have the important advantage of revealing intact proteins with potentially critical post-translational modifications for reaction with autoantibodies in patient sera.

\section{Uncovering antigens using protein microarrays}

Protein microarrays have been developed as an alternative or complement to 2D Western blots, because of higher sensitivity and potential for higher throughput and standardization. For the detection of autoantibodies, fractions from cell lysates are printed onto nitrocellulose-coated slides, probed with patient sera and then fluorescent substrate-conjugated anti-human IgG antibody, and measured for fluorescent inten- 
Table 1

Reported tumor antigens recognized by autoantibodies in various cancer patients' sera, identified by proteomic methods

\begin{tabular}{|c|c|c|c|c|}
\hline Antigens & Types of tumor & Sera positive (rate) & Method & Ref. \\
\hline \multirow[t]{2}{*}{ Annexin I } & Lung adeno & $12 / 30(40 \%)$ & $2 \mathrm{D}-\mathrm{W}^{*}$ & [2] \\
\hline & Lung squamous & $3 / 18(17 \%)$ & & \\
\hline \multirow[t]{2}{*}{ Annexin II } & Lung adeno & $11 / 30(37 \%)$ & $2 \mathrm{D}-\mathrm{W}$ & {$[2]$} \\
\hline & Lung squamous & $4 / 18(22 \%)$ & & \\
\hline PGP9.5 & Lung adeno & $6 / 40(15 \%)$ & $2 \mathrm{D}-\mathrm{W}$ & [3] \\
\hline Vimentin & Pancreas adeno & $16 / 36(44 \%)$ & $2 \mathrm{D}-\mathrm{W}$ & [9] \\
\hline Calreticulin & Pancreas adeno & $21 / 36(58 \%)$ & 2D-W & [10] \\
\hline UCH-L3 & Colon & $19 / 43(44 \%)$ & Protein microarray & [18] \\
\hline$\beta$-tubulin I and III & Neuroblastoma & $11 / 23(48 \%)$ & $2 \mathrm{D}-\mathrm{W}$ & {$[20]$} \\
\hline RS/DJ-1 & Breast & $13 / 30(43 \%)$ & $2 \mathrm{D}-\mathrm{W}$ & [15] \\
\hline Calreticulin & Liver HCC & $10 / 37(27 \%)$ & $2 \mathrm{D}-\mathrm{W}$ & [14] \\
\hline$\beta$-tubulin & “ & $9 / 37(24 \%)$ & $2 \mathrm{D}-\mathrm{W}$ & [14] \\
\hline HSP60 & “ & $5 / 37(14 \%)$ & 2D-W & [14] \\
\hline Cytokeratin 18 & “ & $5 / 37(14 \%)$ & $2 \mathrm{D}-\mathrm{W}$ & [14] \\
\hline Cytokeratin 8 & “ & $4 / 37(11 \%)$ & $2 \mathrm{D}-\mathrm{W}$ & [14] \\
\hline Creatine kinase B & “ & $5 / 37(14 \%)$ & $2 \mathrm{D}-\mathrm{W}$ & [14] \\
\hline \multicolumn{5}{|l|}{ F1-ATP } \\
\hline synthetase $\alpha$ subunit & “ & $4 / 37(11 \%)$ & $2 \mathrm{D}-\mathrm{W}$ & [14] \\
\hline NDPKA & “ & $5 / 37(14 \%)$ & $2 \mathrm{D}-\mathrm{W}$ & [14] \\
\hline Carbonic anhydrase I & Kidney RCC & $3 / 11(27 \%)$ & $2 \mathrm{D}-\mathrm{W}$ & [12] \\
\hline SM22- $\alpha$ & “ & $5 / 11(45 \%)$ & $2 \mathrm{D}-\mathrm{W}$ & [12] \\
\hline
\end{tabular}

*2-dimensional polyacrylamide gel electrophoresis, followed by Western blot.

sity [16,21]. This method is based on liquid-phase fractionation of intact proteins, which can maintain the native state of proteins. Multiple fractions showing significantly higher signals with sera from pulmonary adenocarcinoma [21] and prostate cancer [1] patients than with sera from persons without cancers were recently identified by this method. With a highly reproducible microarray system, 63 of 1840 fractions (3.4\%) derived from an A549 lung cancer cell line lysate, prepared by Rotofor isoelectric focusing (20 fractions) followed by reversed phase HPLC ( 92 fractions), showed statistically higher reactivity with 18 cancer patient sera than 15 normal sera, indicating that these fractions could contain the corresponding antigens of lung cancer-specific autoantibodies [21]. A similar microarray system showed 38 of 1760 fractions (20 Rotofor fractions followed by reversed phase HPLC fractions into 88 fractions each) from a $\mathrm{LnCaP}$ prostate cancer cell line lysate had statistically higher reactivity with 25 prostate cancer patient sera than 25 normal sera; 2 of the 1760 showed higher reactivity with normal sera [1]. Mass spectrometry of the fractions is needed to identify the protein antigens. Among 39 of 1760 arrayed fractions from a LoVo colon adenocarcinoma cell line lysate which showed enhanced reactivity with sera from patients with colon cancer relative to sera from controls, specific autoantibody to ubiquitin C-terminal hydrolase isozyme 3 (UCH-L3) was identified by this method [18].
Analogous studies of autoantibodies with antigen arrays have generated extensive findings for autoimmune disorders [11,22]; at least fourteen autoimmune disorders have one or more clinically-used diagnostic autoantibodies [22].

Protein microarrays also are useful to detect antigens when the slide is coated with specific antibodies (antibody microarray) [6]. Samples can be labeled directly with signal-generating radioisotope, fluorescent or chemiluminescent substrates, followed by incubation with antibody-coated slides and detection by appropriate methods. Two different substrates can be used simultaneously for a test sample and a reference sample as an internal control. Samples can be labeled indirectly using a hapten like biotin, followed by fluorophore-labeled streptoavidin to increase sensitivity and to reduce the interference of immunoreaction by fluorophore directly attached to proteins. Sandwich assays using two different antibodies are also available, though it is challenging to optimize conditions for two different kinds of antibodies. Antibody microarrays that measure many proteins in cancer patient sera are potentially better suited for early detection, staging and classification by multivariate statistical methods than relying on a single cancer biomarker [6].

\section{Multiplex ELISA approaches}

Enzyme-linked immuno-sorbent assays can be arrayed in microplates with multiple wells. Multiplex 
ELISA is highly-suited for automation with 96-, 384-, or 1536-well microplate systems for high-throughput screening (HTS) [5]. We are performing 384-well multiplex ELISA to identify autoantibodies to cancers in patient sera. A 1536-well format is now commercially available; it may be more readily applied to homogenous assays like fluorescent polarization (FP), which do not need a washing step, than to conventional ELISA, which requires a reliable washing step. The same liquid-phase fractions from tumor or cell line lysates prepared for microarrays can be employed in this method. The solid phase chemistry of the microplate is one of the important factors in ELISA; a high protein-binding surface (for example, treated by irradiation of gamma ray) is suitable for multiplex ELISA. Microplate wells are coated with fractions from cell lysates, probed with patient sera and then with signalgenerating substrate-conjugated anti-human IgG antibody, and measured for signal with colorimetric, chemiluminescent, or fluorescent assays [24]. Protein concentration for coating wells is a key parameter; usually protein concentration of $5-15 \mu \mathrm{g} / \mathrm{ml}$ is used, though sometimes much higher concentration might be needed to obtain enough signal for the detection of autoantibody. Robotic liquid handling systems can be used for the distribution of sera and dispensing of reagent, followed by automated microplate washing of the multiplex ELISA. Miniaturization of microplate wells requires more precise liquid handling, more reproducibility of microplate, and faster movement to reduce evaporation of sample or reagents [24].

The advantages of ELISA are the potential for automation, the flexibility of experimental protocols, the familiarity of reference laboratories with multiplex ELISA, and the feasibility of quantitating autoantibody titers. Current work in this laboratory is aimed at optimizing the antigen concentrations required for reliable detection of autoantibodies.

\section{Conclusion}

A growing number of cancer-specific autoantibodies discovered with proteomics approaches have been reported as cancer biomarker candidates. Validation of these markers notably as currently in progress under the National Cancer Institute Early Detection Research Network (EDRN) protocols, may yield combinations of biomarkers for further large-scale validation studies to determine sensitivity, specificity, and positive predictive value in real-world screening scenarios. Hope- fully, these biomarkers will make feasible the diagnosis of common cancers, much earlier in the development of the tumors, when therapy can be curative, thus reducing their mortality.

\section{Acknowledgement}

This work is supported in part by grant MEDC-238 from the Michigan Life Sciences Corridor.

\section{References}

[1] K. Bouwman et al., Microarrays of tumor cell derived proteins uncover a distinct pattern of prostate cancer serum immunoreactivity, Proteomics 3 (2003), 2200-2207.

[2] F. Brichory et al., An immune response manifested by the common occurrence of annexins I and II autoantibodies and high circulating levels of IL-6 in lung cancer, Proc Natl Acad Sci USA 98 (2001), 9824-9829.

[3] F. Brichory et al., Proteomics-based identification of protein gene product 9.5 as a tumor antigen that induces a humoral immune response in lung cancer, Cancer Res 61 (2001), 79087912.

[4] A.O. Gure et al., Human lung cancer antigens recognized by autologous antibodies: definition of a novel cDNA derived from the tumor suppressor gene locus on chromosome $3 \mathrm{p} 21.3$, Cancer Res 58 (1998), 1034-1041.

[5] P. Gwynne, Time, money, and assay development, Drug Discovery and Development 6 (2003), 38-42.

[6] B.B. Haab, Methods and applications of antibody microarrays in cancer research, Proteomics 3 (2003), 2116-2122.

[7] S. Hanash, Disease proteomics, Nature 422 (2003), 226-232.

[8] S. Hanash, Harnessing immunity for cancer marker discovery, Nat Biotechnol 21 (2003), 37-38.

[9] S. Hong et al., Identification of vimentin as a tumor antigen that induces an antibody response in pancreatic cancer, submitted.

[10] S. Hong et al., An autoantibody medintel immune response to calreticulin isoforms in pancretic cancer, Cancer Res, in press.

[11] T.O. Joos et al., A microarray enzyme-linked immunosorbent assay for autoimmune diagnostics, Electrophoresis 21 (2000), 2641-2650.

[12] C.S. Klade et al., Identification of tumor antigens in renal cell carcinoma by serological proteome analysis, Proteomics 1 (2001), 890-898.

[13] R. Kobayashi, A proteomics approach to find a new breast cancer-specific antigenic marker, Clin Cancer Res 7 (2001), 3325-3327.

[14] F. Le Naour et al., A distinct repertoire of autoantibodies in hepatocellular carcinoma identified by proteomic analysis, Mol Cell Proteomics 1 (2002), 197-203.

[15] F. Le Naour et al., Proteomics-based identification of RS/DJ1 as a novel circulating tumor antigen in breast cancer, Clin Cancer Res 7 (2001), 3328-3335.

[16] J. Madoz-Gurpide et al., Protein based microarrays: a tool for probing the proteome of cancer cells and tissues, Proteomics 1 (2001), 1279-1287.

[17] P.J. Mintz et al., Fingerprinting the circulating repertoire of antibodies from cancer patients, Nat Biotechnol 21 (2003), 57-63. 
[18] M.J. Nam et al., Molecular profiling of the immune response in colon cancer using protein microarrays: occurrence of autoantibodies to ubiquitin C-terminal hydrolase L3, Proteomics 3 (2003), 2108-2115.

[19] L.J. Old et al., New paths in human cancer serology, $J$ Exp Med 187 (1998), 1163-1167.

[20] L. Prasannan et al., Identification of beta-tubulin isoforms as tumor antigens in neuroblastoma, Clin Cancer Res 6 (2000), 3949-3956.

[21] J. Qiu et al., Development of natural protein microarrays for diagnosing cancer based on an antibody response to tumor antigens, J Proteome Res 3 (2004), 261-267.

[22] W.H. Robinson et al., Protein arrays for autoantibody profiling and fine-specificity mapping, Proteomics 3 (2003), 20772084.

[23] T. Soussi, The humoral response to the tumor-suppressor geneproduct p53 in human cancer: implications for diagnosis and therapy, Immunol Today 17 (1996), 354-356.

[24] J. Wolcke et al., Miniaturized HTS technologies - uHTS, Drug Discov Today 6 (2001), 637-646. 


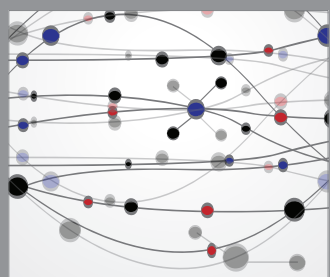

The Scientific World Journal
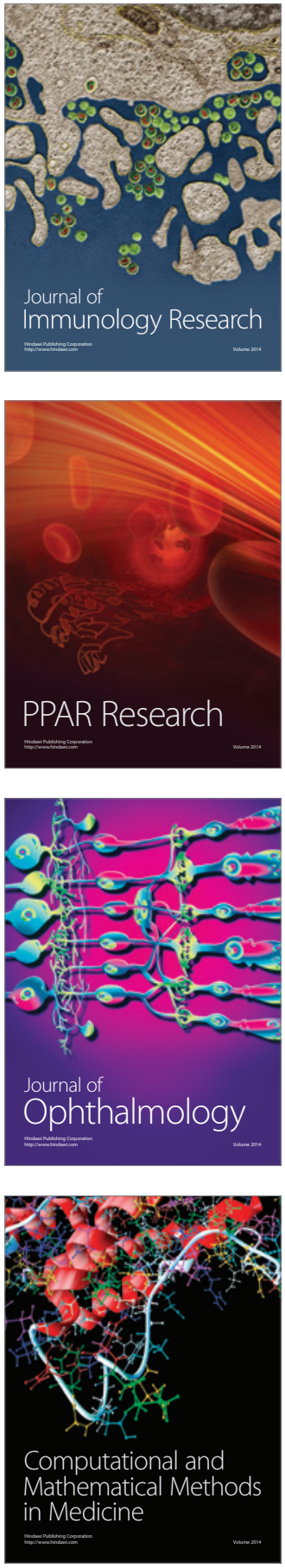

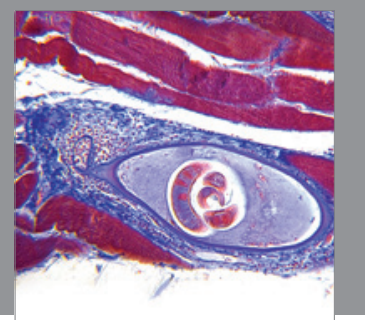

Gastroenterology

Research and Practice
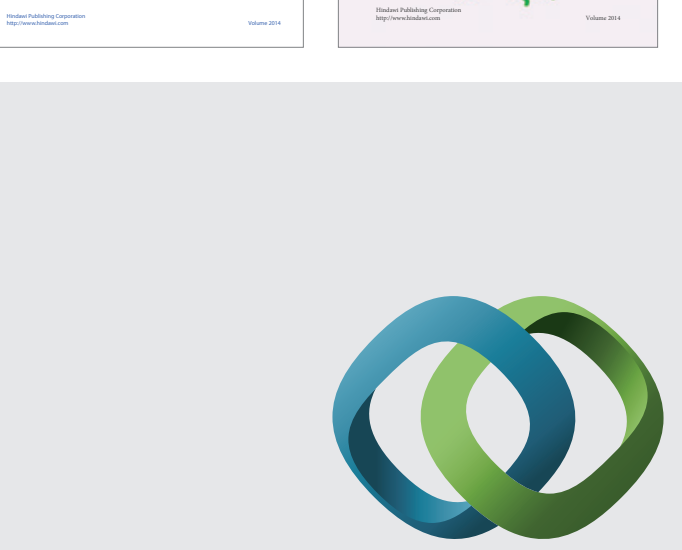

\section{Hindawi}

Submit your manuscripts at

http://www.hindawi.com
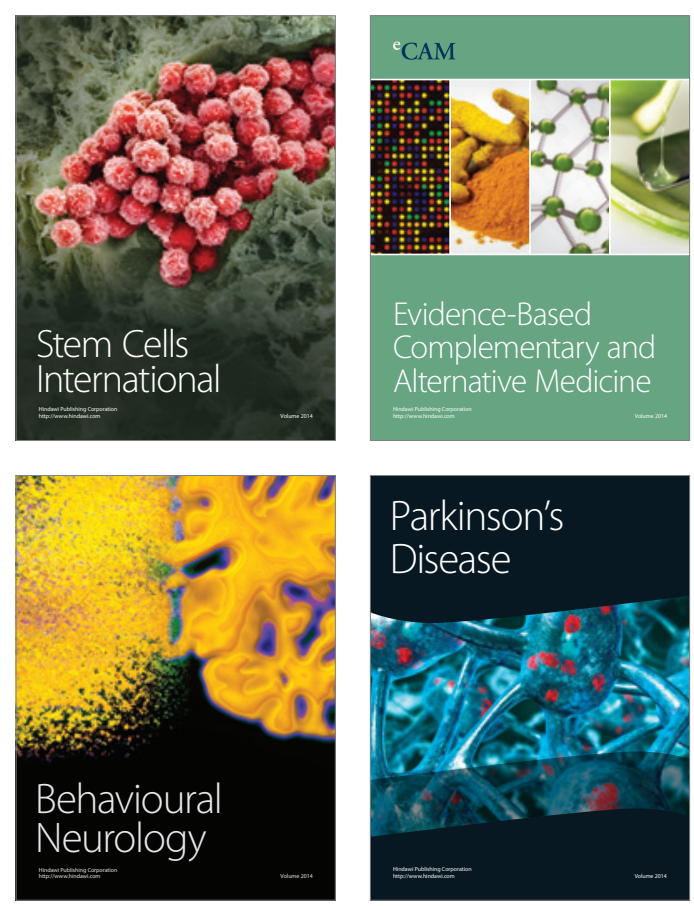

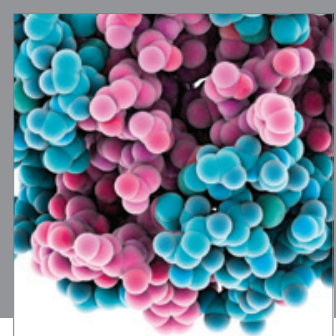

Journal of
Diabetes Research

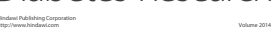

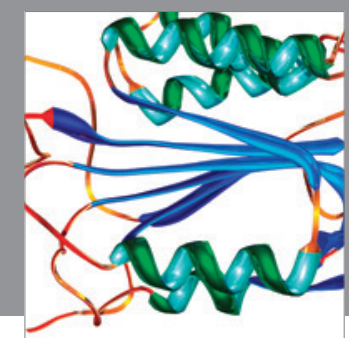

Disease Markers
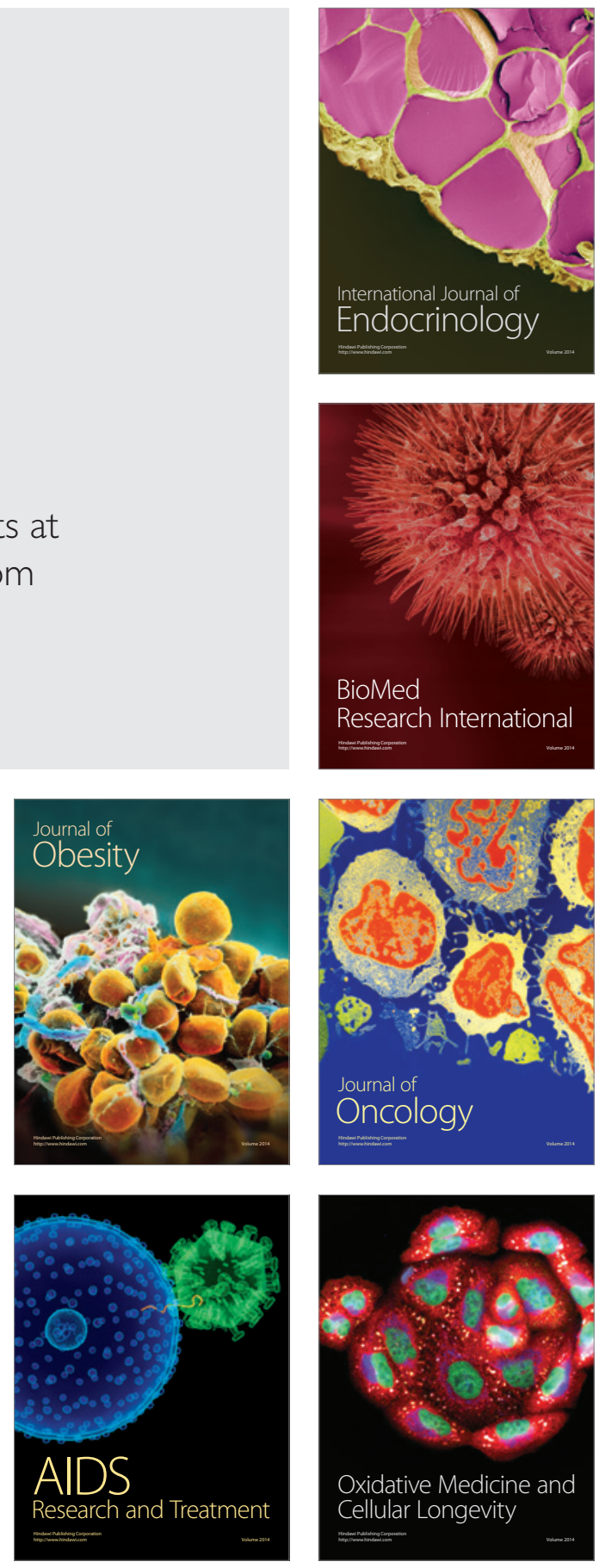